\title{
Prevención familiar del consumo de drogas en Europa: una revisión crítica de los programas contenidos en EDDRA
}

\section{Family prevention of drug use in Europe: a critical review of EDDRA programs}

Daniel Lloret Irles*, José Pedro Espada Sánchez* , Victor Cabrera Perona*, Gregor Burkhart**
* Universidad Miguel Hernández

** European Monitoring Centre for Drug and Drug-addictions.

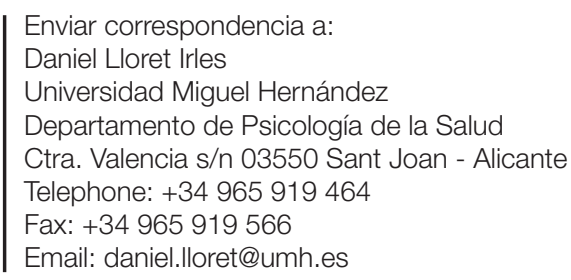

Email: daniel.Iloret@umh.es

\section{Resumen}

En la adolescencia temprana, la familia es uno de los principales agentes de riesgo/protección del inicio del consumo de drogas. Su influencia en la adquisición de hábitos de salud le convierte en diana preferente de intervenciones preventivas. El objetivo es realizar un análisis comparado de los programas de prevención familiar europeos. Se analizaron 85 programas de prevención familiar contenidos en la base de datos EDDRA (Exchange on Drug Demand Reduction Action), perteneciente al Observatorio Europeo de las Drogas y Toxicomanías. Fueron analizados todos los programas incluidos en la base, que abarca el periodo 1998-2011. El 53\% de los programas proceden de sólo 4 países (España, Alemania, Portugal e Irlanda). El 89\% son inespecíficos o de promoción de la salud. Proporcionar información sobre drogas es el principal objetivo que persiguen los programas. Algo más de la mitad de los programas (59.7\%) informan haber realizado evaluación de resultados. El 70.59\% de los programas no plantea entre sus objetivos ninguno de los factores de riesgo propuestos en las principales teorías. A pesar del sólido marco teórico y de la evidencia empírica sobre los factores de riesgo familiares, los programas de prevención presentan una gran dispersión en sus objetivos, técnicas y componentes. El grado de adecuación a las teorías es muy bajo y la evaluación sigue siendo la asignatura pendiente. Se plantean críticas sobre el funcionamiento de la base EDDRA. recibido: Noviembre 2012 aceptado: Mayo 2013

\section{Abstract}

In early adolescence, family is one of the main risk/protection agent for drug misuse. Its influence on health habits acquisition makes family a key target for prevention programs. The aim is to conduct a comparative analysis of European family prevention programs. For this purpose, 85 programs were reviewed. Programs were retrieved from EMCDDA database EDDRA (Exchange on Drug Demand Reduction Actions). No time period was delimited; hence time span was 1998-2011. 53\% of all programs came from 4 countries (Ireland, Germany, Portugal and Spain). 89\% were unspecific drug prevention or health promotion programs. Providing information about drugs is the main objective pursued by the majority of the programs. Over half the programs (59.7\%) report having undergone an outcome evaluation process. Regarding the program objectives, $70.59 \%$ do not address any of the risk factors proposed by the main theories in international literature. Despite the solid theoretical framework that supports coherent evidence about family risk factors, prevention programs' objectives and components have a low theoretical compliance rate and the level of evaluation continues to be a challenge. Some aspects of the EDDRA functioning are reviewed.

Keywords: Prevention, Family, Europe, Review, EDDRA 
L os datos de la última encuesta ESPAD, European School Survey Project on Alcohol and Other Drugs, (Hibell et al., 2012) revelan que en Europa el 54\% de los jóvenes de 15 años ha probado el tabaco al menos una vez en la vida, y más de la mitad de estos (el 51,85\%) informaron haber fumado en los últimos 30 días. En cuanto al consumo de alcohol, su arraigada aceptación cultural hace que el $57 \%$ de los jóvenes europeos lo hayan consumido en los últimos 30 días, y que el $12 \%$ haya consumido alcohol antes de los 13 años. El 39\% informa haber bebido en exceso al menos una vez en los últimos 30 dias, y el 14\% reconoce haberlo hecho como mínimo tres veces. La tercera droga más consumida es el cannabis, cuya prevalencia de consumo en los últimos doce meses es del 13\%, y el $7 \%$ declara haberlo usado en los últimos 30 días. Ellickson, Tucker, Klein y Saner (2004) sostienen que consumir marihuana antes de los 15 años aumenta significativamente el riesgo del posterior abuso, no sólo de marihuana sino también del resto de drogas.

En la temprana edad en la que se producen los primeros consumos, el menor se socializa bajo la influencia de cuatro principales agentes: la familia, los amigos, la escuela y los medios de comunicación (Bauman, Carver y Gleiter, 2001; Becoña, 2002; de Vries, Engels, Kremers, Wetzels y Mudde, 2003; Kristjansson, Sigfusdottir, James, Allegrante y Helgason, 2010; Oetting y Donnermeyer, 1998). Entre estos agentes, la familia destaca por su particular ascendencia en la adquisición de nuevas conductas y formación de actitudes de los menores (Bricker, Peterson, Sarason, Andersen y Rajan, 2007; Maccoby, 1992; Maccoby y Martin, 1983). Algunos factores familiares han sido considerados generales por ser comunes a diversas conductas problemáticas: el conflicto familiar (Lam, Solmeyer y McHale, 2012; Mullineaux, Deater-Deckard, Petrill y Thompson, 2009), la escasa o nula supervisión (Cottrell et al., 2003;
DiClemente et al., 2001; Jimenez Iglesias, 2009; Mott, Crowe, Richardson y Flay, 1999; Webb, Bray, Getz y Adams, 2002), la baja cohesión familiar (Paxton, Valois y Drane, 2007; Wagner et al. 2010) o las deficiencias en comunicación (Demant y Ravn, 2013; Kaplow, Curran y Dodge, 2002). Por otra parte, se consideran factores de riesgo específicos: el consumo por otros miembros de la familia, en especial por el padre o la madre (Becoña et al., 2012; Brook et al., 2010; Vermeulen-Smit et al., 2012) y la actitud favorable, o al menos no contraria, al consumo (Foxcroft y Lowe, 1997; Lloret, Segura y Carratalá, 2008; Wen et al., 2005).

La influencia de la familia en el consumo de drogas de los hijos ha sido recogida en diversas teorías. Este cuerpo teórico ha impulsado una fértil investigación que identifica determinadas características familiares, factores de riesgo, que se asocian con el consumo de drogas de los hijos. (Tabla1)

La evidencia en torno al papel de la familia, como agente modulador de las conductas de riesgo de los hijos, constituye la base en la que debería descansar el diseño de los programas de prevención del consumo de drogas. Por consiguiente, se espera que la reducción de los factores de riesgo y el refuerzo de los factores de protección que encuentran su fundamento en la teoría, y han sido respaldados por los resultados de investigación, constituyan los objetivos de los programas. Así mismo, la necesaria evaluación de la eficacia de los programas requiere la inclusión de indicadores que permitan monitorear, de forma objetiva y fiable, el impacto de la intervención en tales factores, y en el propio consumo.

Visto así, los programas de prevención son instrumentos dinámicos sometidos a una monitorización y evaluación que posibilita un proceso de mejora continua y su adaptación a los cambios sociales. Los resultados de las investigaciones sobre la

Tabla 1

Presencia de factores de riesgo en los modelos teóricos

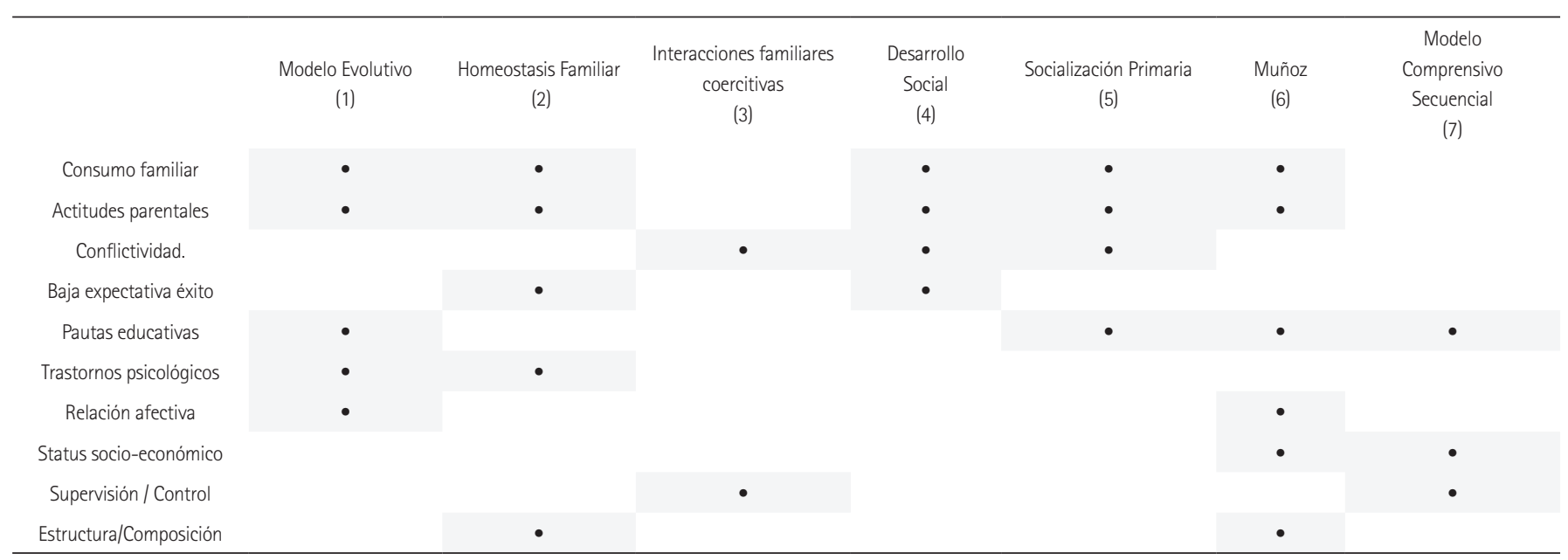

(1) Kandel, 1980; Kandel y Davies, 1992.(2) Stanton et al. 1978; Stanton, 1979, 1980; Stanton y Shadish, 1997

(3) Patterson, 1979; Patterson, 1982; Patterson y Dishion, 1985;

(4) Catalano y Hawkins, 1996; Farrington y Hawkins, 1991; Hawkins y Weis, 1985; Hawkins, Catalano y Miller, 1992

(5) Oetting y Donnermeyer, 1998; Oetting, Deffenbacher y Donnermeyer, 1998a, 1998b; 1998c

(6) Muñoz-Rivas, Graña y Cruzado, 2000.

(7) Becoña, 1999, pp. 265-275 
eficacia y la eficiencia de los programas de prevención son la principal fuente de información para la mejora de los mismos. Cada vez que un programa se aplica, se debe recoger datos del proceso de implementación y de los resultados, que se contrastan con los objetivos a alcanzar, de manera que los desarrolladores puedan reajustar los componentes del programa en función de las discrepancias halladas. La aplicación de este procedimiento genera ciclos continuos de "implementaciónevaluación-mejora-implementación" a los que se someten los programas y que les suponen un continuo cambio (Brotherhood y Sumnall, 2011).

En Europa se cuenta con un abundante bagaje de intervenciones preventivas del consumo de drogas que operan en el ámbito familiar. Aunque gran parte de esta experiencia es aún inaccesible por no haber sido debidamente analizada y publicada, durante la última década la cultura de evaluación se va gradualmente incorporando, y se están realizando esfuerzos dirigidos a revisar y clasificar la vasta oferta de programas de intervención para el ámbito familiar (Espada y Mendez, 2002; Horgan, 2011; Foxcroft y Tsertsvadze, 2011; Smit, Verdurmen, Monshouwer y Smit, 2008).

\section{Registros de programas de prevención eficaces.}

Con el fin de difundir las mejores prácticas y facilitar el acceso a los programas preventivos con probada calidad, diversas instituciones han puesto en marcha sistemas online de selección y registro de programas. Estos sistemas consisten en colecciones de fichas técnicas con la información detallada de los programas, que permiten localizar el programa más idóneo para el tipo de prevención deseada.

En Estados Unidos el Substance Abuse and Mental Health Services Administration (SAMHSA) gestiona el Registro Nacional de Programas y Prácticas basados en la Evidencia (NREPP: http://www.nrepp.samhsa.gov/). Contiene programas de tratamiento y prevención de las enfermedades mentales y abuso de drogas que han sido evaluados y clasificados por revisores independientes. NREPP ofrece una descripción de cada programa, sus objetivos, los resultados obtenidos, el soporte empírico y datos de contacto del responsable. NREPP facilita información sobre la calidad de la evidencia científica que avala los resultados de cada programa, y también informa sobre el tipo de materiales utilizados y de la necesidad de formación previa para la implementación del programa. El registro de nuevos programas parte de la iniciativa voluntaria del responsable del programa, quien solicita su incorporación. En consecuencia, es plausible pensar que alguna intervención, aun gozando de buena calidad, no haya sido remitida a NREPP, por lo que no puede ser considerado un registro exhaustivo de los programa de prevención y prevención que tienen calidad.

El Canadian Best Practice Portal (http://cbpp-pcpe.phacaspc.gc.ca/), gestionado por la Agencia de Salud Pública, es una iniciativa similar, que contiene únicamente programas e intervenciones de carácter comunitario que persiguen la prevención de enfermedades crónicas, incluido el consumo de drogas, y la promoción de la salud. Al igual que en NREPP, cualquiera puede proponer un programa, que será revisado por un panel de expertos que garantiza que el programa cuenta con apoyo científico.

En Australia, la Australian Drug Foundation, ofrece la Australian Drug Information Network -ADIN-(http://www.adin. com.au/content.asp?Document_ID=39), una base de datos de información sobre drogas y recursos para la prevención y el tratamiento del consumo de drogas. Su objetivo es facilitar el acceso a recursos seleccionados según criterios de calidad.

A nivel europeo, el Observatorio Europeo sobre Drogas y Toxicomanías (EMCDDA) ha desarrollado la base EDDRA (Exchange on Drug Demand Reduction Action), un proyecto en el que participan 30 países europeos. Consiste en un sistema de información cuyo fin es facilitar el acceso a los recursos de buenas prácticas preventivas. Cuenta con la colaboración de una red de corresponsales nacionales que se encargan de localizar y seleccionar nuevos programas, y remitir un cuestionario normalizado para cada programa. Los programas seleccionados deben haber sido al menos sometidos a evaluación de proceso, y son calificados de acuerdo a un sistema de puntuación que asigna un 1 a los programas con menor evidencia (generalmente sólo evaluación de proceso), mientras que el nivel más alto - 3 - son por lo general los programas que han sido objeto de estudios controlados aleatorizados.

Los resultados de las búsquedas en la base EDDRA ofrecen fichas estándar que describen las características de los programas incluidos. La información relativa a cada programa incluye el marco teórico, un análisis del contexto, los objetivos, las acciones, el resultado de las evaluaciones, los indicadores de resultado, los agentes de cambio, etc. Actualmente, EDDRA contiene más de 400 registros de intervenciones de carácter preventivo y asistencial clasificados en cinco ámbitos principales: prevención, tratamiento, reintegración social, reducción del daño e intervenciones en el sistema judicial. El ámbito prevención se clasifica a su vez en ambiental, universal, selectiva o indicada. Además del ámbito, los programas se agrupan por distintos criterios, como el tipo de abordaje, la población diana, el tipo de evaluación aplicado o el tipo de sustancia objeto de la prevención.

Este trabajo tiene como objetivo describir las principales características de los programas de prevención familiar del consumo de drogas mediante la revisión de los contenidos de la base de datos EDDRA. Se pretende conocer su nivel de evaluación y analizar el grado de coherencia de los objetivos de los programas con los factores de riesgo avalados por la evidencia.

\section{Método}

Para la identificación de los programas de prevención, se utilizó el motor de búsqueda de la base EDDRA. Todos los programas que respondian al descriptor Prevention en "type of intervention" y "family" en Target group" o "setting" o "Type of approach" fueron recuperados.

No se acotaron periodos, por lo que el periodo analizado abarcó desde 1998 hasta 2011. Inicialmente fueron recuperadas 87 fichas, que fueron revisadas por dos expertos que trabajaron independientemente. En una primera revisión se 
identificaron dos programas de prevención cuyo ámbito principal no era la familia, por lo que la muestra final quedó compuesta por 85 fichas de programas de prevención.

Dos revisores expertos analizaron los programas través de la información contenida en EDDRA, y los clasificaron según el ámbito geográfico, el tipo de prevención según la taxonomía propuesta por el Instituto de Medicina (Mrazek y Haggerty, 1994), en universal, selectiva o indicada, la población destinataria, el objetivo general, los objetivos específicos, el tipo de evaluación y el estado de la misma. Para el análisis de la evaluación se consideraron los siguientes tipos: proceso, resultados e impacto. La evaluación de proceso permite conocer si el programa se ha desarrollado tal como se planteó, evidenciando los obstáculos que han podido surgir. La evaluación de resultados analiza el grado de consecución de los objetivos planteados. La evaluación de resultados determina, si los cambios buscados en la población diana se deben a la intervención. En definitiva, mide la eficacia y la eficiencia del programa. Por último, la evaluación de impacto que indica el efecto del programa en macroindicadores de abuso a nivel comunitario (Leukefeld y Bukoski, 1991) y evalúa los efectos más allá de los objetivos propuestos por el programa.

Las posibles discordancias se resolvieron de común acuerdo con la intervención de un tercer experto. En el mes de marzo 2011, se realizó la búsqueda en la Base EDDRA, cada una de las fichas fue descargada y extraída la información estándar, tabulándose en una hoja de cálculo. Posteriormente, fueron realizados análisis descriptivos de frecuencias.

\section{Resultados}

\section{Análisis descriptivo de los programas de preven- ción familiar incluidos en EDDRA}

\section{Distribución geográfica}

La distribución de programas según su país de origen revela amplias diferencias entre las aportaciones de cada país. Como muestra la tabla 2, cuatro países: Portugal (15.3\%), España (14\%), Irlanda (13\%) y Alemania (10.6\%) cubren más del $50 \%$ de los programas rescatados. Otros países destacados en el número de programas aportados son Finlandia, Austria, Grecia, y Holanda. Finalmente un grupo de países se hallan menos representados, aportando sólo uno o dos programas: Bélgica, Dinamarca, Polonia, Suecia, Croacia, Italia, UK, Luxemburgo y Noruega.

\section{Población diana}

El 56.4\% de los programas analizados tiene como población diana la familia en su totalidad, el $16.5 \%$ se dirige a niños/jóvenes, el 5.9\% a adultos, el 7\% restante a población general. Es de resaltar que el $14 \%$ de los programas analizados no identificaba su población diana.
Tabla 2

Características de los programas preventivos

\begin{tabular}{|c|c|c|}
\hline & $\%$ & $n$ \\
\hline \multicolumn{3}{|l|}{ Pais de origen } \\
\hline Portugal & 15.3 & 13 \\
\hline España & 14.1 & 12 \\
\hline Irlanda & 12.9 & 11 \\
\hline Alemania & 10.6 & 9 \\
\hline Finlandia & 8.2 & 7 \\
\hline Austria & 5.9 & 5 \\
\hline Grecia & 4.7 & 4 \\
\hline Holanda & 4.7 & 4 \\
\hline Bélgica & 3.5 & 3 \\
\hline Dinamarca & 3.5 & 3 \\
\hline Polonia & 3.5 & 3 \\
\hline Suecia & 3.5 & 3 \\
\hline Italia & 2.4 & 2 \\
\hline Reino Unido & 2.4 & 2 \\
\hline Croacia & 1.2 & 1 \\
\hline Luxemburgo & 1.2 & 1 \\
\hline Noruega & 1.2 & 1 \\
\hline Comisión Europea & 1.2 & 1 \\
\hline \multicolumn{3}{|l|}{ Población diana } \\
\hline Familia & 56.4 & 48 \\
\hline Niños/Adolescentes & 16.5 & 14 \\
\hline Adultos & 5.9 & 5 \\
\hline General & 7.1 & 6 \\
\hline Sin definir & 14.1 & 12 \\
\hline \multicolumn{3}{|l|}{ Tipo de prevención } \\
\hline Universal & 70.5 & 60 \\
\hline Selectiva & 20.0 & 17 \\
\hline Indicada & 7.1 & 6 \\
\hline Sin definir & 2.4 & 2 \\
\hline \multicolumn{3}{|c|}{ Sustancias a las que se dirige el programa } \\
\hline Tabaco+Alcohol+Cannabis & 3.5 & 3 \\
\hline Cannabis & 3.5 & 3 \\
\hline Alcohol & 2.4 & 2 \\
\hline Tabaco & 1.2 & 1 \\
\hline Sin definir & 89.4 & 76 \\
\hline
\end{tabular}

\section{Tipo de prevención}

El 70.5\% de los programas realiza prevención universal, dirigida a una población que no presenta un nivel de riesgo preocupante. Un 20\% de los programas efectúa prevención selectiva, centrada en grupos en los que el nivel de riesgo es superior a la media de la población general, y tan sólo un 7.1\% prevención indicada, es decir familias con alto riesgo o consumidores. El restante $2.4 \%$ corresponde a programas sin definir.

\section{Sustancias tratadas}

La gran mayoría de los programas analizados $(89,4 \%)$ actúan sobre el riesgo de consumo de cualquier sustancia. El objetivo de estos programas se centra en poten- 
Tabla 3

Objetivos de los programas preventivos (Frecuencias)

\begin{tabular}{|c|c|c|}
\hline & $\%$ & $\mathrm{n}$ \\
\hline \multicolumn{3}{|l|}{ Objetivos generales } \\
\hline Desarrollo de habilidades de comunicación o sociales & 13.1 & 14 \\
\hline Formación (a padres, profesores). & 13.1 & 14 \\
\hline Información & 10.3 & 11 \\
\hline Fomentar factores de prevención & 10.3 & 11 \\
\hline Desarrollo de habilidades para padres. & 6.5 & 7 \\
\hline Prevención o reducción del consumo & 5.6 & 6 \\
\hline Compartir experiencias (redes de padres) & 4.7 & 5 \\
\hline Hábitos saludables & 4.7 & 5 \\
\hline Prevenir situaciones de riesgo & 4.7 & 5 \\
\hline Proveer de ayuda & 2.8 & 3 \\
\hline Manejo del autocontrol & 2.8 & 3 \\
\hline Cooperación entre profesionales y padres & 2.8 & 3 \\
\hline Sensibilización & 2.8 & 3 \\
\hline Promoción de la salud & 2.8 & 3 \\
\hline Detección temprana & 2.8 & 3 \\
\hline Desarrollo psicosocial & 1.9 & 2 \\
\hline Orientación y apoyo. Counselling. & 1.9 & 2 \\
\hline Ofrecer alternativas & 1.9 & 2 \\
\hline Uso de las tecnologías & 1.9 & 2 \\
\hline Reducir comportamientos patológicos & 0.9 & 1 \\
\hline Afrontamiento & 0.9 & 1 \\
\hline Retrasar la edad de inicio de consumo & 0.9 & 1 \\
\hline \multicolumn{3}{|l|}{ Objetivos especificos } \\
\hline \multicolumn{3}{|l|}{ Objetivos especificos sobre drogas } \\
\hline Proporcionar información & 29.4 & 25 \\
\hline Cambio de actitudes & 9.4 & 8 \\
\hline Proporcionar counselling & 9.4 & 8 \\
\hline Fomentar factores de protección & 7.1 & 6 \\
\hline Reducir consumo/intención de consumo & 7.1 & 6 \\
\hline Prevenir situaciones de riesgo & 4.7 & 4 \\
\hline Compartir experiencias & 4.7 & 4 \\
\hline Retrasar la edad de inicio de consumo & 3.5 & 3 \\
\hline Detección precoz & 2.4 & 2 \\
\hline \multicolumn{3}{|l|}{ Estilos parentales/educativos } \\
\hline Educación de padres & 8.2 & 7 \\
\hline Estilos parentales & 5.9 & 5 \\
\hline Normas y limites & 5.9 & 5 \\
\hline Dinámicas familiares & 1.2 & 1 \\
\hline \multicolumn{3}{|l|}{ Habilidades sociales } \\
\hline Habilidades sociales & 18.8 & 16 \\
\hline Comunicación & 17.6 & 15 \\
\hline Resolución de conflictos & 3.5 & 3 \\
\hline Resistencia a la presión social & 3.5 & 3 \\
\hline Rehabilitación social & 3.5 & 3 \\
\hline Prevención de comportamientos antisociales & 2.4 & 2 \\
\hline Tiempo libre & 1.2 & 1 \\
\hline \multicolumn{3}{|l|}{ Objetivos individuales } \\
\hline Autoimagen/autocontrol/emociones positivas & 7,1 & 6 \\
\hline Resolución de problemas & 7,1 & 6 \\
\hline Hábitos saludables & 4.7 & 4 \\
\hline Estrategias de afrontamiento & 4.7 & 4 \\
\hline Autoestima en niños/padres & 4.7 & 4 \\
\hline Establecimiento de metas & 1.2 & 1 \\
\hline No agrupados en los anteriores & 36.5 & 31 \\
\hline
\end{tabular}

ciar factores de protección o reducir factores de riesgo de carácter general, tanto del individuo como de la interacción individuo/entorno. El 11\% restante son programas dirigidos a la prevención específica de drogas, y más concretamente tabaco+alcohol+cannabis (3.5\%), cannabis (3.5\%), alcohol $(2.4 \%)$ y tabaco $(1.2 \%)$.

\section{Objetivos generales}

Un alto porcentaje de los programas presentó un solo objetivo general. En cuanto a los objetivos más habituales entre los programas analizados, destacan: formar a padres (13.1\%), desarrollar habilidades o competencias sociales y/o de comunicación (13.1\%), informar (10.3\%) y fomentar factores de prevención (10.3\%). La distribución total de los objetivos generales puede verse en la tabla 3.

\section{Objetivos específicos}

Los objetivos específicos de los programas evaluados, han sido agrupados en cuatro grandes categorías (Tabla 3). En el primer grupo "objetivos específicos sobre drogas" (64.7\%) incluye programas que ofrecen información sobre las consecuencias del consumo, modifican la actitud y/o la conducta de consumo. "Proporcionar información" (29.4\%) es el objetivo más perseguido. En el otro extremo, destaca que la detección precoz es un objetivo que sólo lo propone un programa (2.4\%) .

El segundo grupo, "estilos educativos" (20\%), consistió entrenamiento de técnicas/habilidades para fomentar el aprendizaje y modificar la conducta de los hijos. Un tercer grupo se denominó "habilidades sociales" (43.5\%), en él se agrupan los objetivos dirigidos al desarrollo de competencias personales para una mejor gestión de la vida social. Se identificaron 7 objetivos diferentes, siendo el más frecuente la adquisición de habilidades sociales.

El grupo de "objetivos individuales" (24.7\%) se centra en el desarrollo de técnicas de autocontrol basadas en el individuo y en la promoción de un autoconcepto positivo. El resto de objetivos no incluidos en los grupos anteriores tuvo una frecuencia de aparición del $36.4 \%$. Todos los programas presentan más de un objetivo específico. No se ha encontrado relación entre el tipo de prevención empleada (universal, selectiva o indicada ) y el objetivo específico perseguido (Tabla 4).

\section{Adecuación de los programas a la evidencia sobre factores de riesgo}

En este apartado se determina el grado de coherencia entre los objetivos de los programas y los factores de riesgo recogidos en los modelos teóricos sobre influencia familiar resumidos en la tabla 1.

De los 85 programas analizados, sólo 25 (29.41\%) incluyeron entre sus objetivos al menos un factor de riesgo de los identificados en las teorías sobre la influencia de la familia en el consumo de drogas de los hijos. Ocho programas (9.4\%) contemplaron la intervención en dos o más factores de riesgo, y tan sólo uno intervino sobre tres factores. El factor más frecuentemente abordado fue la "comunicación entre padres/ madres e hijo/as", tratado por 15 programas (17.6\%). La "actitud de los padres hacia las drogas y el propio consumo por los padres" fue abordado por el $9.4 \%$ de los programas. Por último, los factores "supervisión y disciplina" y "estilo educativo" se trabajaron en cinco programas (5.88\%). El resto de factores identificados en las teorías, no han sido propuestos como objetivos en los programas revisados. 
Tabla 4

Relación entre los objetivos específicos y el tipo de prevención

\begin{tabular}{|c|c|c|c|c|c|c|c|c|}
\hline \multirow[b]{2}{*}{ Objetivo especifico } & \multicolumn{8}{|c|}{ Tipo de prevención } \\
\hline & & & Universal & Selectiva & Indicada & Total & c & $\mathrm{p}$-valor \\
\hline \multirow[t]{5}{*}{ Drogas } & & & & & & & 0.116 & 0.570 \\
\hline & \multirow{2}{*}{ NO } & $n$ & 26 & 8 & 4 & 38 & & \\
\hline & & $\%$ & $68.4 \%$ & $21.1 \%$ & $10.5 \%$ & $100.0 \%$ & & \\
\hline & \multirow{2}{*}{$\mathrm{SI}$} & n & 33 & 9 & 2 & 44 & & \\
\hline & & $\%$ & $75.0 \%$ & $20.5 \%$ & $4.5 \%$ & $100.0 \%$ & & \\
\hline \multirow[t]{5}{*}{ Estilo Parental } & & & & & & & 0.034 & 0.954 \\
\hline & \multirow{2}{*}{ NO } & $n$ & 47 & 14 & 5 & 66 & & \\
\hline & & $\%$ & $71.2 \%$ & $21.2 \%$ & $7.6 \%$ & $100 \%$ & & \\
\hline & \multirow{2}{*}{ SI } & $n$ & 12 & 3 & 1 & 16 & & \\
\hline & & $\%$ & $75.0 \%$ & $20.7 \%$ & $7.3 \%$ & $100 \%$ & & \\
\hline \multirow[t]{5}{*}{ Habilidades Sociales } & & & & & & & 0.071 & 0.812 \\
\hline & \multirow{2}{*}{ NO } & $n$ & 35 & 11 & 3 & 49 & & \\
\hline & & $\%$ & $71.4 \%$ & $22.4 \%$ & $6.1 \%$ & $100 \%$ & & \\
\hline & $S$ & $n$ & 24 & 6 & 3 & 33 & & \\
\hline & $\pi$ & $\%$ & $72,7 \%$ & $18,2 \%$ & $7,3 \%$ & $100 \%$ & & \\
\hline \multirow[t]{5}{*}{ Individual } & & & & & & & 0.090 & 0.722 \\
\hline & \multirow{2}{*}{ NO } & $n$ & 45 & 12 & 5 & 62 & & \\
\hline & & $\%$ & $72.6 \%$ & $19.4 \%$ & $8.1 \%$ & $100 \%$ & & \\
\hline & \multirow{2}{*}{$\mathrm{SI}$} & $n$ & 12 & 5 & 1 & 18 & & \\
\hline & & $\%$ & $66.7 \%$ & $27.8 \%$ & $5.6 \%$ & $100 \%$ & & \\
\hline
\end{tabular}

Tabla 5

Relación entre el tipo de evaluador y la modalidad de Evaluación

\begin{tabular}{|c|c|c|c|c|c|c|}
\hline & & \multicolumn{3}{|c|}{ Modalidad Evaluación } & \multirow[b]{2}{*}{$x^{2}$} & \multirow[b]{2}{*}{$\mathrm{p}$-valor } \\
\hline & & Proceso & Resultado & Total & & \\
\hline Tipo de evaluador & & & & & 2.376 & 0.305 \\
\hline \multirow[t]{3}{*}{ Interno } & Recuento & 18 & 19 & 37 & & \\
\hline & \% dentro de Tipo_Evaluador & $48,6 \%$ & $51,4 \%$ & $100,0 \%$ & & \\
\hline & \% dentro de Modalidad Evaluación & $62,1 \%$ & $44,2 \%$ & $51,4 \%$ & & \\
\hline \multirow[t]{3}{*}{ Externo } & Recuento & 8 & 19 & 27 & & \\
\hline & \% dentro de Tipo_Evaluador & $29,6 \%$ & $70,4 \%$ & $100,0 \%$ & & \\
\hline & \% dentro de Modalidad Evaluación & $27,6 \%$ & $44,2 \%$ & $37,5 \%$ & & \\
\hline \multirow[t]{3}{*}{ Mixto } & Recuento & 3 & 5 & 8 & & \\
\hline & \% dentro de Tipo_Evaluador & $37,5 \%$ & $62,5 \%$ & $100,0 \%$ & & \\
\hline & \% dentro de Modalidad Evaluación & $10,3 \%$ & $11,6 \%$ & $11,1 \%$ & & \\
\hline \multirow[t]{3}{*}{ Total } & Recuento & 29 & 43 & 72 & & \\
\hline & \% dentro de Tipo_Evaluador & $40,3 \%$ & $59,7 \%$ & $100,0 \%$ & & \\
\hline & \% dentro de Modalidad Evaluación & $100,0 \%$ & $100,0 \%$ & $100,0 \%$ & & \\
\hline
\end{tabular}




\section{Evaluación de los programas preventivos}

Para EDDRA, la evaluación es uno de los pilares de buenas prácticas y posibilita la toma de decisiones sobre las intervenciones realizadas y la comunicación fiable de los resultados del programa, por lo que representa uno de los elementos clave para la admisión de un programa. De los 85 programas analizados, el 40.3\% refirió evaluación solamente del proceso, mientras que el 59.7\% informó haber evaluado los resultados. En este segundo grupo el $11.8 \%$ evaluó proceso y resultados, y el $11.8 \%$ realizó las tres evaluaciones: proceso, resultados e impacto. En referencia al tipo de evaluador (interno/externo), de los 72 programas que informaron sobre el evaluador, el 51.4\% fueron evaluados por los propios responsables, evaluación interna. Algo más de un tercio de los programas (37.5\%) fueron evaluados únicamente por expertos externos, y el $11.1 \%$ fue sometido a evaluación mixta. No se encontraron diferencias significativas en la relación tipo de evaluador y modalidad de evaluación (Tabla 5).

\section{Discusión}

Los objetivos del presente trabajo son describir las principales características de los programas de prevención familiar incluidos en la base EDDRA, y analizar el grado de coherencia de sus objetivos con los factores de riesgo y protección que propugnan las principales teorias.

Por lo general, se observa una baja coherencia entre los objetivos de los programas y los factores de riesgo establecidos en la literatura científica. Teniendo en cuenta que riesgo familiar se define como la configuración de ciertas caracteristicas o dinámicas que interactúan entre ellas, potenciándose y aumentando la probabilidad de consumo, es aconsejable que un mismo programa incluya el mayor número posible de factores. Sin embargo, ningún programa incluye entre sus objetivos más de tres factores de riesgo, sólo un programa alcanzó los tres.

Ciertos aspectos del funcionamiento de EDDRA suponen limitaciones a la hora de generalizar los resultados del presente trabajo al conjunto de la prevención familiar en Europa. En primer lugar, se exige un grado mínimo de evaluación para ser admitido en EDDRA. Aunque este criterio supone a priori una ventaja, limita el número de programas incluidos en relación a los programas disponibles en cada pais. No se dispone del listado exhaustivo de programas que se están implementando, no obstante es de esperar que el número de programas familiares sin evaluación alguna sea probablemente bastante más elevado.

El número de programas por país incluidos en EDDRA podria indicar, no tanto la cantidad de programas que actualmente se aplican en cada país, sino a la actividad de los Puntos Focales Nacionales (PFN) y la cultura de conceptualización y evaluación de las intervenciones preventivas. Aunque el objetivo del estudio no es realizar un análisis comparativo entre paises, sino describir los programas preventivos realizados en
Europa, si sería deseable un mayor número de programas aportados por cada pais.

Un segundo aspecto a tener en cuenta es el procedimiento de actualización de la base de datos EDDRA. La identificación de nuevos programas, su selección y la cumplimentación de las fichas son tareas encomendadas a los PFN. La respuesta de los puntos nacionales, no es homogénea por diferentes motivos. No existe un procedimiento estandarizado de recogida de información que garantice la identificación de programas, su sistemática revisión y la adopción de los mismos criterios de selección. La eliminación de los programas obsoletos y la inclusión de nuevos, depende en gran medida del nivel de actividad del PFN y de sus conocimientos de las redes de prevención en el respectivo pais. En definitiva, no siempre se garantiza que la selección de programas se ajuste a los mismos criterios en todos los países, pero se ha asumido que los programas evaluados y con un modelo lógico se encuentran por lo general en EDDRA, pues estos programas más evolucionados tienden a ser más conocidos en los círculos profesionales de prevención.

En tercer lugar, llama la atención la dispersión encontrada en la categorización de los objetivos planteados por los programas. A falta de un sistema cerrado de categorización de objetivos, los responsables de completar las fichas definen los objetivos de cada programa según su propia opinión o atendiendo a la información facilitada por los diseñadores del programa. Si bien la posibilidad de una opinión abierta enriquece y matiza las particularidades de cada programa, dificulta a su vez la clasificación y por lo tanto la comparabilidad entre programas. Con el fin de poder clasificar el mayor número posible de objetivos, se identificaron todos los objetivos que proponen los programas y posteriormente se agruparon en una clasificación ad hoc. Dicha clasificación plantea categorías no excluyentes. Algunos objetivos presentan un cierto grado de solapamiento, mientras otros hacen referencia a las actividades a realizar, más que a los resultados a obtener.

En cuarto y último lugar, se debe tener en cuenta que las estructuras administrativas de dichos PFN se ven periódicamente afectadas por circunstancias politicas, tales como variaciones presupuestarias, competencia con otras prioridades, inestabilidad laboral... etc. que se traducen en fluctuaciones en el funcionamiento interno y altibajos en la productividad.

Estos argumentos podrían explicar la fuerte dispersión numérica entre los paises, España o Irlanda aportan 5 veces más programas que Holanda, Italia o el Reino Unido. Especialmente en el caso del Reino Unido puede haber insuficiencias en las entradas proporcionadas por el PFN, pues existen varios programas para familias evaluados en el pais (Lloyd, Wollny, White, Gowland y Purdon, 2011) que nunca han sido introducidos en EDDRA.

Este análisis de la prevención familiar en Europa evidencia que incluso los programas más selectos, considerados "leading edge" por su inclusión en EDDRA, en su mayoría carecen de unas bases teóricas y científicas sólidas. Asumiendo las citadas limitaciones del presente trabajo, las insuficiencias conceptuales de los programas contenidos en EDDRA parecen reflejar el estado de la prevención familiar en Europa. Para la mayoría de los paises europeos (con excepción de Reino Unido o Noruega) 
se puede deducir que los programas que no están insertados en EDDRA cuentan con un menor soporte teórico y evidencia experimental. Este hecho también se refleja en el catálogo de programas de prevención basados en pruebas de UNODC (2010). Con la excepción del Strengthening Families Programme (que sí está en EDDRA) los programas ahí listados no se implementan en Europa, o sólo se hace a escala experimental.

\section{Conflicto de intereses}

El presente trabajo no ha recibido ninguna financiación. Los autores declaran que no tienen conflicto de interés.

\section{Referencias}

Bauman, K. E., Carver, K. y Gleiter, K. (2001). Trends in parent and friend influence during adolescence: the case of adolescent cigarette smoking. Addictive Behaviours, 26, 349-361. Doi: 10.1016/S03064603(00)00110-6

Becoña, E. (1999). Bases teóricas que sustentan los programas de prevención de drogas. Madrid: Plan Nacional sobre Drogas.

Becoña, E. (2002) Factores de riesgo y protección familiar para el uso de drogas. En J. R. Fernández-Hermida y R. Secades-Villa (Eds.), Intervención familiar en la prevención de las drogodependencias. Madrid: Plan Nacional sobre Drogas.

Becoña, E., Martínez, U., Calafat, A., Juan, M., Duch, M.A. y FernándezHermida, J. R. (2012). ¿Cómo influye la desorganización familiar en el consumo de drogas de los hijos? Una revisión. Adicciones, 24, 253-268.

Bricker, J. B., Peterson, A. V.Jr., Sarason, I. G., Andersen, M. R. y Rajan, K. B.(2007). Changes in the influence of parents' and close friends' smoking on adolescent smoking transitions. Addictive Behaviours, 32, 740-757. Doi: 10.1016/j.addbeh.2006.06.020

Brotherhood, A. y Sumnall, H. (2011) European drug prevention quality standards. A manual for prevention professionals. Lisbon: European Monitoring Center for Drug and Drug Addiction.

Brook, J. S., Balka, E. B., Crossman, A. M., Dermatis, H., Galanter, M. y Brook, D. W. (2010). The relationship between parental alcohol use, early and late adolescent alcohol use, and young adult psychological symptoms: A longitudinal study. American Journal on Addictions, 19, 534-542. Doi: 10.1111/j.1521-0391.2010.00083.x

Catalano, R. F. y Hawkins, J. D. (1996). The social development model: A theory of antisocial behavior. En J. D. Hawkins (ed), Delinquency and Crime: Current Theories. New York: Cambridge University Press.

Cottrell, L., Li, X., Harris, C., D'Alessandri, D., Atkins, M., Richardson, B. y Stanton, B. (2003). Parent and adolescent perceptions of parental monitoring and adolescent risk involvement. Parenting: Science \& Practice, 3, 179-195.

Demant, J. y Ravn, S. (2013). Communicating trust between parents and their children: A case study of adolescents' alcohol use in Denmark. Journal of Adolescent Research, 28, 325-347. Doi: $10.1177 / 0743558413477198$

de Vries, H., Engels, R., Kremers, S., Wetzels, J. y Mudde, A. (2003). Parents' and friends' smoking status as predictors of smoking onset: findings from six European countries. Health Education Research, 18, 627-636. Doi: $10.1093 /$ her/cyg032

DiClemente, R. J., Wingood, G. M., Crosby, R., Sionean, C., Cobb, B. K. y Harrington, K.(2001). Parental monitoring: Association with adolescents' risk behaviors. Pediatrics, 107, 1363-1368. Doi: 10.1542/ peds.107.6.1363

Ellickson, P. L., Tucker, J. S., Klein, D. J. y Saner, H. (2004). Antecedents and outcomes of marijuana use initiation during adolescence. Preventive Medicine, 39, 976- 984. Doi: 10.1016/j.ypmed.2004.04.013

European Monitoring Centre for Drugs and Drug Addiction (2010). Prevention and Evaluation Resources Kit (PERK). Lisboa: Author.

Espada, J. P. y Méndez, F. X. (2002). Intervención familiar en la prevención del abuso de drogas. En J. R. Fernández-Hermida y R. Secades-Villa (Eds.), Intervención familiar en la prevención del abuso de drogas. Madrid: Plan Nacional sobre Drogas.

Farrington, D. P. y Hawkins, J. D. (1991). Predicting participation, early onset, and later persistence in officially recorded offending. Criminal Behavior and Mental Health 1,1-33.

Foxcroft, D. y Lowe, G. (1997). Adolescents' alcohol use and misuse: the socializing influence of perceived family life. Drugs: education, prevention and policy, 4, 215-229.

Foxcroft, D. y Tsertsvadze, A. (2011). Universal family-based prevention programs for alcohol misuse in young people (Review). The Cochrane Library, 9. Wiley \& Sons.

Hawkins, J. D. y Weis, J. G. (1985). The social development model: An integrated approach to delinquency prevention. Journal of Primary Prevention, 6, 73-97.

Hawkins, J. D., Catalano, R. F. y Miller, J. L. (1992). Risk and protective factors for alcohol and other drug problems in adolescence and early adulthood: Implications for substance abuse prevention. Psychological Bulletin, 112, 64-105.

Hibell, B., Guttormsson, U., Ahlström, S., Balakireva, O., Bjarnason, T., Kokkevi, A. y Kraus L. (2012). The 2011 ESPAD Report. The Swedish Council for Information on Alcohol and other Drugs (CAN). Stockholm: ESPAD.

Horgan, J. (2011). Parental Substance Misuse: Addressing its Impact on Children A Review of the Literature. Dublin: National Advisory Committee on Drugs.

Jimenez Iglesias, A. (2009). El significado de la monitorización familiar en el desarrollo adolescente (consumo de sustancias y bienestar psicológico). Madrid: Centro de Investigación y Estudios de Familia (CIFAM).

Kandel, D. B. (1980). Developmental stages in adolescent drug involvement. En D. J. Lettieri, M. Sayers y H. W. Pearson (Eds.), Theories on drug abuse. Selected contemporary perspectives (pp. 120-127). Rockville, MD: NIDA Research Monograph, 30.

Kandel, D. B. y Davies, M. (1992). Progression to regular marijuana involvement: Phenomenology and risk factors for near-daily use. En M. Glantz y R. Pickens (Eds.), Vulnerability to drug abuse (pp. 211-253). Washington, DC: American Psychological Association.

Kaplow, J. B., Curran, P. J. y Dodge, K. A. (2002). Child, Parent, and Peer Predictors of Early-Onset Substance Use: A Multisite Longitudinal Study. Journal of Abnormal Child Psychology, 30, 199-216. Doi: 10.1023/A:1015183927979 
Kristjansson, A. L., Sigfusdottir, I. D., James, J. E., Allegrante, J. P. y Helgason, A. R. (2010). Perceived parental reactions and peer respect as predictors of adolescent cigarette smoking and alcohol use. Addictive Behaviours, 35, 256-259. Doi: 10.1016/j.addbeh.2009.10.002

Lam, C. B., Solmeyer, A. R. y McHale, S. M. (2012). Sibling differences in parent-child conflict and risky behavior: A three-wave longitudinal study. Journal of Family Psychology, 26, 523-531. Doi: 10.1037| a0029083

Leukefeld, C. G. y Bukoski, W. J. (1991). An Introduction to Drug Abuse Prevention Intervention Research: Methodological Issues. En C. G. Leukefeld, y W. J. Bukoski (Eds.), Drug Abuse Prevention Intervention Research: Methodological Issues. NIDA Research Monograph, 107. Rockville: NIDA.

Lloret, D., Segura, M. y Carratalá, E. (2008). Relaciones y reacciones familiares y consumo de alcohol y tabaco en adolescentes en población rural. Health and Addictions, 8, 119-135.

Lloyd, Ch., Wollny, I., White, C., Gowland, S. y Purdon, S. (2011). Monitoring and evaluation of family intervention services and projects between February 2007 and March 2011. Research Report Department for Education FE-RR174. London: U.K. Government.

Maccoby, E. E. (1992). The role of parents in the socialization of children: An historic overview. Developmental Psychology, 28, 1006-1017.

Maccoby, E. E. y Martin, J. A. (1983). Socializations in the contexts of the family: Parent child interactions. En P. H. Mussen (Ed.), Handbook of child psychology. Vol. 4. Socialization, personality, and social development. Nueva York: Wiley.

Mott, J. A., Crowe, P. A., Richardson, J. y Flay, B. (1999). After-school supervision and adolescent cigarette smoking: Contributions of the setting and intensity of after-school self-care. Journal of Behavioral Medicine, 22, 35-58. Doi: 10.1023/A:1018747602026

Mrazek, P. J. y Haggerty, R. J. (Ed). (1994). Reducing risks for mental disorders: Frontiers for preventive intervention research. Institute of Medicine. Washington DC: National Academy.

Muñoz-Rivas, M. J., Graña, J. L. y Cruzado, J. A. (2000). Factores de riesgo en drogodependencias: Consumo de drogas en adolescentes. Madrid: Sociedad Española de Psicología Clínica, Legal y Forense.

Mullineaux, P. Y., Deater-Deckard, K., Petrill, S. A., y Thompson, L. A. (2009). Parenting and child behaviour problems: A longitudinal analysis of non-shared environment. Infant and Child Development, 18, 133-148. doi:10.1002/icd.593

Oetting, E. R. y Donnermeyer, J. F. (1998). Primary socialization theory: The etiology of drug use and deviance I. Substance Use \& Misuse, 33, 9951026.

Oetting, E. R., Deffenbacher, J. L. y Donnermeyer, J. F. (1998a). Primary socialization theory: The role played by personal traits in the etiology of drug use and deviance. II. Substance Use \& Misuse, 33, 1337-1366.

Detting, E. R., Donnermeyer, J. F. y Deffenbacher, J. L. (1998b). Primary socialization theory: The influence of the community on drug use and deviance. III. Substance Use \& Misuse, 33, 1629-1665.

Oetting, E. R., Donnermeyer, J. F., Trimble, J. E. y Beauvais, F. (1998c). Primary socialization theory: Culture, ethnicity, and cultural identification. The links between culture and substance use. IV. Substance Use \& Misuse, 33, 2075- 2107
Patterson, G.R. (1979). A performance theory for coercive family interactions. En R. Cairns (Ed.), Social Interaction: Methods, Analysis, and Illustration (pp. 119-162). Hillsdale, NJ: Lawrence Erlbaum Associates, Inc.

Patterson, G. R. (1982). Coercive Family Process. Eugene, OR: Castalia.

Patterson, G. R. y Dishion, T. J. (1985). Contributions of families and peers to delinquency. Criminology, 23, 63-79.

Paxton, R. J., Valois, R. F. y Drane, J. W. (2007). Is there a relationship between family structure and substance use among public middle school students? Journal of Child and Family Studies, 16, 593-605. Doi: 10.1007/s10826-006-9109-y

Peterson, P. L., Hawkins, J. D. y Catalano, R. F. (1992).. Evaluating comprensive community drug risk reduction interventions. Design challenges and recommenations. Evaluation Review, 16, 579-602.

Smit, E., Verdurmen, J., Monshouwer, K. y Smit, F. (2008). Family interventions and their effect on adolescent alcohol use in general populations: a meta-analysis of randomised controlled trials. Drug and Alcohol Dependence, 97, 195-206. Doi: 10.1016/j.drugalcdep.2008.03.032

Stanton, M. D., Todd, T. C., Heard, D. B., Kirschner, S., Kleiman, J. I., Mowatt, D. T. y Riley, P. (1978). Heroin addiction as a family phenomenon: A new conceptual model. American Journal of Drug and Alcohol Abuse, 5, 125-150.

Stanton, M. D. (1979). Drugs and the family. Marriage and Family Review, 2,1-10.

Stanton, M. D. (1980). Aspects of the family and drug abuse. En: Ellis, B. (Ed.), Drug Abuse from the Family Perspective: Coping Is a Family Affair. Rockville, MD.: National Institute on Drug Abuse.

Stanton, M. D. y Shadish, W. (1997). Outcome, attrition, and family-couples treatment of drug abuse: A meta-analysis and review of the controlled, comparative studies. Psychological Bulletin, 122, 170-191.

United Nations Office on Drugs and Crime. UNDOC (2010). Compilation of Evidence-Based Family Skills Training Programmes. New York; NY: UNDOC.

Vermeulen-Smit, E., Koning, I.M., Verdurmen, J.E.E., Van der Vorst, H., Engels, R.C.M.E. y Vollebergh, W.A.M. (2012). The influence of paternal and maternal drinking patterns within two-partner families on the initiation and development of adolescent drinking. Addictive Behaviors, 37, 1248-1256. Doi: 10.1016/j.addbeh.2012.06.005

Wagner, K. D., Ritt-Olson, A., Chou, C.-P., Pokhrel, P., Duan, L., BaezcondeGarbanati, L. y Soto, D. W. (2010). Associations between family structure, family functioning, and substance use among hispanic/ latino adolescents. Psychology of Addictive Behaviors, 24, 98-108. Doi: $10.1037 / a 0018497$

Webb, J. A., Bray, J. H., Getz, J. G. y Adams, G. (2002). Gender, perceived parental monitoring, and behavioral adjustment: Influences on adolescent alcohol use. American Journal of Orthopsychiatry, 72 (3), 392-400. Doi: 10.1037/0002-9432.72.3.392

Wen, C. P., Tsai, S. P., Cheng, T. Y., Hsu, C. C., Chen, T. y Lin, H. S. (2005). Role of parents and peers in influencing the smoking status of high school students in Taiwan. Tobacco Control, 14, i10-i15. Doi: 10.1136/ tc.2003.005637. 